\title{
Self-consistent model of electron drift mode turbulence
}

\author{
Z H A N N A N. A N D R USHCHENK $\mathrm{O}^{1}$, \\ MARTIN JUCKER ${ }^{2}$ and VLADIMIR P. PAVLENK ${ }^{3}$ \\ ${ }^{1}$ Swedish University of Agricultural Sciences, SE-750 07 Uppsala, Sweden \\ ${ }^{2}$ École Polytechnique Fédérale de Lausanne, Association EURATOM-Confédération \\ Suisse, CH-1015 Lausanne, Switzerland \\ ${ }^{3}$ Uppsala University and EURATOM-VR Fusion Association, SE-751 20 Uppsala, Sweden \\ (vladimir.pavlenko@astro.uu.se)
}

(Received 20 July 2006, accepted in revised form 29 December 2006)

\begin{abstract}
The nonlinear dynamics of magnetic electron drift mode turbulence are outlined and the generation of large-scale magnetic structures in a non-uniform magnetized plasma by turbulent Reynolds stress is demonstrated. The loop-back of large-scale flows on the microturbulence is elucidated and the modulation of the electron drift mode turbulence spectrum in a medium with slowly varying parameters is presented. The wave kinetic equation in the presence of large-scale flows is derived and it can be seen that the small-scale turbulence and the large-scale structures form a self-regulating system. Finally, it is shown by the use of quasilinear theory that the shearing of microturbulence by the flows can be described by a diffusion equation in $\mathbf{k}$-space and the corresponding diffusion coefficients are calculated.
\end{abstract}

\section{Introduction}

The nonlinear study of turbulence in plasma physics is strongly related to the appearance of structures with higher symmetry, in contrast to the random underlying turbulence. Usual drift wave turbulence is known to generate fluid motions with additional symmetry [1], frequently referred to as flows, which are commonly divided into zonal flows and streamers [2]. Zonal flows are defined here as symmetric structures with a finite scale in the direction of plasma inhomogeneity, significantly larger than the scale of the underlying small-scale turbulence [3,4]. Streamers are convective cells which complement zonal flows, in that they have short proportions in the direction of translation symmetry, i.e. perpendicular to plasma gradients, and are elongated structures in the direction of plasma inhomogeneity $[5,6]$. This elongation along one defined direction is attributed to an additional symmetry of large-scale flows compared with the randomly distributed small-scale fluctuations.

The mechanisms for the generation of such flows have been extensively studied in many astrophysical, geophysical and laboratory settings. For instance, the generation of zonal flows by Rossby waves in the atmosphere has recently been studied in [7] and these waves are known to have a significant influence on larger scales, for example the Jet Stream in the atmosphere and the Gulf Stream in oceanography [8]. In addition, zonal flows have for a long time been observed in experimental and 
numerical simulations of rotating neutral fluids and have been invoked to explain the striped atmospheres of giant planets. Regions of alternating azimuthal velocity have been found on Saturn and Jupiter by the Voyager spacecraft. In recent years, the study of zonal flows and streamers and their interactions with drift wave turbulence [9] has become an important part of theoretical plasma physics, most of all because of its assumed relation to anomalous transport $[10,11]$.

Transport and amplification properties of large-scale magnetic fields are an area of active investigation, mostly because of their importance in a range of physical phenomena. One impressive effect of large, strong magnetic fields is the release of high-energy bursts in solar flares [12]. These bursts are believed to occur as a result of the reconnection of magnetic field lines, which one attempts to understand through turbulent magnetic field diffusivity, relating directly to the question of the transport of large-scale magnetic fields in a turbulent environment [13-16]. Other possible mechanisms of reconnection may be via magnetic streamers [17], which are radially elongated magnetic structures. The investigation of magnetic streamers is motivated by the similarity to the radially extended convective cells in drift wave turbulence, which represent a mechanism for describing the often observed bursty or intermittent transport in simulations and experiments. Furthermore, through their radial extension, magnetic streamers have the potential for greatly increased thermal transport, and, together with zonal magnetic fields (poloidally extended structures), are candidates for the significant increase in electron energy transport [18].

On the other hand, since the end of the $1970 \mathrm{~s}$, experiments have demonstrated that strong quasi-steady magnetic fields are created in laser-produced plasmas [19]. This was an important result as it had often been assumed that the absence of magnetic field effects, which greatly affect heat transport, was a desirable feature of laser-produced plasmas [20]. These measurements showed clearly that strong magnetic fields can be generated even in magnetized plasmas [21]. Closer investigations revealed that these magnetic fields oscillate with a typical frequency between the ion and the electron plasma frequencies, and are fed by density and temperature gradients through the first-order baroclinic vector [22].

In order to investigate the generation of strong magnetic fields within the aforementioned frequency range, we will in this article develop the nonlinear theory of magnetic electron drift wave turbulence in an unmagnetized inhomogeneous plasma. This theory is a two-field theory, in contrast to the theory of electrostatic drift wave turbulence.

The coupling of small-scale fluctuations to mean flow can be described by the kinetic equation for wave packets [23] and corresponds to the conservation of an action-like invariant of the wave turbulence with slowly varying parameters due to the mean sheared flow. However, the conserved action-like quantity ('pseudoaction') is different from the usual definition of the wave action, since, as has been noted in [24] and [25], the standard action variables used to describe the selfinteraction between small-scale fluctuations without the shear flow are modified by the flow and may not be suitable.

Here we develop a self-consistent theory of magnetic electron drift mode turbulence. The model should not only include the generation of strong, large-scale magnetic fields by microturbulence but also the back reaction of these structures on the small-scale drift wave turbulence. The linear theory of magnetic electron drift modes, developed for an unmagnetized non-uniform plasma on the basis of 
fluid models [26-28], shows that unstable motion is fed by inhomogeneities in electron density and temperature [29]. In the low-frequency limit (compared with the ion plasma frequency), electron inertia is usually neglected, whereas in the highfrequency limit it must be included and the ion motion can be neglected. We will restrict ourselves to this limit. The mode does not depend on collisional effects or heat flux $[27,28]$. In the collisionless regime, the source of instability is the baroclinic vector in the electron fluid, which gives rise to finite vorticity. The electron inertia manifests itself in the electron fluid vorticity and together with the temperature perturbation is essential for these modes. In this approximation nonlinear effects can be very important, since the generated magnetic field itself evolves through an explicitly nonlinear equation. The basic nonlinear system of equations has been derived in [30] and different nonlinear effects have been studied in [31-33].

In the present paper we focus on the derivation of the wave kinetic equation for magnetic electron drift mode turbulence. We establish the generation of largescale flows by microscale turbulence via Reynolds stress and their counter-effect on the small-scale wave turbulence. This leads us to the evolution equation for the wave spectrum, the wave kinetic equation. Finally, shearing of small-scale turbulence by large-scale structures is shown. The rest of the paper is organized as follows. In the next section we give a brief reminder of the model equations for the magnetic electron drift modes. In Sec. 3.1, the excitation of large-scale flows is examined and the mechanism of large-scale flow generation via Reynolds stress is clearly shown. In Sec. 3.2, the influence of the excited large-scale structures on the parent small-scale turbulence is investigated and we sort out the evolution of the wave amplitude due to interactions between small-scale turbulence and largescale flows. Furthermore, in Sec. 3.3, we show how to describe the small-scale structures evolving in a medium with slowly changing variables and we introduce a pseudo-action which leads directly to the wave kinetic equation. Then, in Sec. 3.4, quasilinear analysis is used to describe the mechanism for the shearing of smallscale turbulence by large-scale flows. In Sec. 4 we provide a short discussion of our results and conclude the paper.

\section{Basic equations}

Magnetic electron drift modes are low-frequency motions fed by inhomogeneities in electron density and temperature. These modes have typical frequencies of the order of $\kappa v_{\mathrm{Te}}$, where $\kappa$ is the inverse characteristic length of the background inhomogeneity and $v_{\mathrm{Te}}$ is the thermal electron velocity. For our description we consider a non-uniform unmagnetized plasma with equilibrium density and temperature of $n_{0}$ and $T_{0}$, respectively. The time scale of interest is in the range given by the inverse ion and inverse electron plasma frequencies. Hence we consider an unpolarized electron fluid and immobile ions. So, the ions play a passive role as a neutralizing background and the dominant role in dynamics is played by the electron species. Therefore, density perturbations can be neglected, i.e. $n$ equals its equilibrium value $n_{0}$, and we require $\omega \gg \omega_{\text {pi }}$, where $\omega_{\text {pi }}$ is the ion plasma frequency.

In order to derive our model equations, we use the momentum equation together with Maxwell's equations and the energy equation [30],

$$
\left(\frac{\partial}{\partial t}+\mathbf{v} \cdot \nabla\right) \mathbf{v}+\frac{e}{m}(\mathbf{E}+\mathbf{v} \times \mathbf{B})+\frac{1}{m n} \nabla\left(n_{0} T\right)=0,
$$




$$
\begin{gathered}
\nabla \times \mathbf{E}=-\frac{\partial \mathbf{B}}{\partial t}, \\
\nabla \times \mathbf{B}=\mu_{0} \mathbf{j}, \\
n_{0}\left(\frac{\partial}{\partial t}+\mathbf{v} \cdot \nabla\right) T+\frac{2}{3} n_{0} T \nabla \cdot \mathbf{V}=0 .
\end{gathered}
$$

We make the standard assumptions that we consider a two-dimensional case in the $x-y$-plane. Then, all quantities are independent of $z$ and the perturbed magnetic field is directed along the $z$-axis. The length scales of the fluctuations are supposed to be much smaller than those of the equilibrium quantities. The temperature will be considered the sum of an equilibrium and a perturbed part, $T_{0}+T$, and the perturbed magnetic field is denoted by $B$. As a last assumption we consider the equilibrium density $n_{0}$ and temperature $T_{0}$ as functions of $x$ only. Taking the curl of the momentum equation one can show, with all the above assumptions, that the basic system of equations describing both the linear and nonlinear properties of magnetic electron drift modes becomes [30]

$$
\begin{gathered}
\frac{\partial T}{\partial t}+\alpha \frac{\partial B}{\partial y}=-\frac{e \lambda^{2}}{m}\{B, T\}, \\
\frac{\partial}{\partial t}\left(B-\lambda^{2} \nabla^{2} B\right)+\beta \frac{\partial T}{\partial y}=\frac{e \lambda^{4}}{m}\left\{B, \nabla^{2} B\right\} .
\end{gathered}
$$

Here $\alpha=\lambda^{2}\left(e T_{0} / m\right)\left(\frac{2}{3} \kappa_{n}-\kappa_{T}\right)$ and $\beta=\kappa_{n} / e . \kappa_{n}=|\nabla \ln n|$ and $\kappa_{T}=\left|\nabla \ln T_{0}\right|$ are the inverse length scales of the density and the temperature inhomogeneities, respectively, and $\lambda=c / \omega_{\text {pe }}$ is the skin depth.

Note that the evolution equation for the magnetic field is nonlinear in $B$. This is intrinsically due to the convective derivative in the electron momentum equation. The order of perturbation of the right-hand side of (2.4a) (representing the baroclinic vorticity source) shows that the perturbed temperature should not be neglected.

Linearizing the evolution equations (2.4) for small perturbations in $B, T \propto$ $\exp (-i \omega t+i \mathbf{k} \cdot \mathbf{r})$, the dispersion relation for magnetic electron drift modes is obtained with the linear wave eigenfrequency $[28,30]$

$$
\omega_{k}=k_{y} \sqrt{\frac{\alpha \beta}{1+k^{2} \lambda^{2}}}
$$

where $\alpha \beta=v_{\mathrm{Te}}^{2} \lambda^{2} \kappa_{n}\left(\frac{2}{3} \kappa_{n}-\kappa_{T}\right)$. There is a purely growing solution for $\kappa_{T}>\frac{2}{3} \kappa_{n}$ and the growth rate vanishes for modes with $k_{y}=0$. Another useful result from the Fourier decomposition in the model equations is that one can find a relation between the magnetic field Fourier amplitudes $B_{k}$ and those of the temperature $T_{k}$, which is

$$
T_{k}=\frac{\alpha k_{y}}{\omega_{k}} B_{k}
$$

\section{The wave kinetic equation}

\subsection{Excitation of large-scale flows}

We have seen that the underlying magnetic electron drift mode turbulence is driven by gradients of temperature and density. As already mentioned, this 
microturbulence can spontaneously generate large-scale flows. During this flow generation, thermodynamic free energy stored in the gradients is converted into flow kinetic energy by fluctuation-induced Reynolds stress and thus these gradients constitute the energy source for the flow. In this section, we focus on the generation of large-scale magnetic flows by turbulent Reynolds stress.

Our approach uses the ansatz of multiple-scale expansion between the spatiotemporal scales of the flows and those of the microturbulence. We thus assume a sufficient spectral gap separating large- and small-scale motions. The temperature and the magnetic field are then decomposed into a large-scale, slowly varying component (denoted with a bar) and a small-scale component, $\bar{T}+\tilde{T}$ and $\bar{B}+\tilde{B}$, respectively. The system of equations (2.4) has a conserved quantity corresponding to the energy integral given by [34]

$$
E=\int\left(\left(\bar{B}^{2}+\tilde{B}^{2}\right)+\lambda^{2}\left((\nabla \bar{B})^{2}+(\nabla \tilde{B})^{2}\right)+\frac{\beta}{\alpha}\left(\bar{T}^{2}+\tilde{T}^{2}\right)\right) d x d y=\text { constant }
$$

In the following sections it is important to note that the conserved total energy contains both small- and large-scale components. This means that the whole wave spectrum and the interactions between different regions of the spectrum have to be included in subsequent considerations.

Having considered the nonlinear model equation, we now want to describe the interaction between small- and large-scale structures within the turbulence. This means that we are going to separate the whole turbulent spectrum into two parts, one describing large-scale structures with a wave vector denoted by $\mathbf{q}$ and the other describing small-scale turbulence with a wave vector denoted by $\mathbf{k}$. We therefore have the relation $|\mathbf{q}| \ll|\mathbf{k}|$. Note that both $\mathbf{q}$ and $\mathbf{k}$ describe the same spectrum but different parts of it.

We now suppose that the amplitudes vary slowly with time owing to wave-wave interactions and apply to the system (2.4) the spatial Fourier transform with $\mathbf{k}$ and $\mathbf{q}$ describing the two different parts of the spectrum:

$$
\begin{aligned}
\left(\begin{array}{c}
B \\
T
\end{array}\right)(\mathbf{r}, t)= & \sum_{k}\left(\begin{array}{c}
B_{k}(t) \\
T_{k}(t)
\end{array}\right) e^{i \mathbf{k} \cdot \mathbf{r}} \quad \text { for small scales } \\
& +\left(\begin{array}{c}
B_{q}(t) \\
T_{q}(t)
\end{array}\right) e^{i \mathbf{q} \cdot \mathbf{r}}+\text { c.c. for large scales. }
\end{aligned}
$$

Using these decompositions, $|\mathbf{k}| \gg|\mathbf{q}|$ and the result from (2.6), we obtain for the $q$ th Fourier components of equations (2.4)

$$
\begin{gathered}
\frac{\partial T_{q}}{\partial t}+i \alpha q_{y} B_{q}=-\frac{e \lambda^{2}}{2 m} \sum_{k}(\mathbf{k} \times \mathbf{q}) \cdot \hat{\mathbf{z}}\left(T_{k} B_{q-k}-T_{q-k} B_{k}\right)=0, \\
\frac{\partial B_{q}}{\partial t}+\frac{i \beta q_{y}}{1+q^{2} \lambda^{2}} T_{q}=-\lambda^{4} \frac{e}{m} \frac{1}{1+q^{2} \lambda^{2}} \sum_{k}(\mathbf{k} \times \mathbf{q}) \cdot \hat{\mathbf{z}}(\mathbf{k} \cdot \mathbf{q}) B_{k} B_{q-k} .
\end{gathered}
$$

Note that in deriving these equations (and in contrast to [34]) we did not assume a potential character of the flow $(\nabla \times \mathbf{E}=0)$. The right-hand side of (3.3a) vanishes with the assumption that $|\mathbf{k}| \gg|\mathbf{q}|$ and for coherent interactions one can approximate $T_{q-k} \sim T_{-k}$ to leading order [34]. This corresponds to neglecting the noise emitted into the flows by the incoherent coupling of magnetic electron drift modes. 
It is seen that a small-scale turbulence can indeed drive large-scale structures characterized by $T_{q}$ and $B_{q}$. For reasons of clarity, we will cease to call these structures flows, but rather fields, since we are not dealing with particles but magnetic fields and temperature 'flows'. The system of equations (3.3) describes the field structures and addresses both the zonal magnetic field and magnetic streamer formation by small-scale magnetic drift turbulence on an equal footing.

In the limit of zonal magnetic fields $(\mathbf{q}=(q, 0,0))$, the above equations yield

$$
\begin{gathered}
\frac{\partial T_{q}}{\partial t}=0 \\
\frac{\partial B_{q}}{\partial t}=\frac{e \lambda^{2}}{m} \frac{q^{2} \lambda^{2}}{1+q^{2} \lambda^{2}} \sum_{k} k_{x} k_{y} B_{k} B_{-k} .
\end{gathered}
$$

It is a direct consequence of these equations that the mean electron temperature associated with zonal fields does not evolve with time.

For magnetic streamers $(\mathbf{q}=(0, q, 0)),(3.4)$ becomes

$$
\begin{gathered}
\frac{\partial T_{q}}{\partial t}+i \alpha q B_{q}=0 \\
\frac{\partial B_{q}}{\partial t}+i \beta \frac{q}{1+q^{2} \lambda^{2}} T_{q}=-\frac{e \lambda^{2}}{m} \frac{q^{2} \lambda^{2}}{1+q^{2} \lambda^{2}} \sum_{k} k_{x} k_{y} B_{k} B_{-k} .
\end{gathered}
$$

A clear difference between the evolution of streamers and zonal fields can immediately be stated: whereas the evolution equations for zonal fields are decoupled and the temperature can even be considered as a constant, the equations for streamers remain coupled and they always have a real frequency in contrast to the zerofrequency zonal fields. Streamers have a linear eigenfrequency and therefore linear instability is possible in this case.

There is, however, an important similarity between the two large-scale structures, in that they are excited by the same small-scale turbulence via the magnetic Reynolds stress $\sum_{k} k_{x} k_{y} B_{k} B_{-k}$. This is of course not unexpected, since the nonlinear excitation of large-scale flows by Reynolds stress is already well established [35]. In order to describe the nonlinear evolution of the total wave spectrum in a selfconsistent way, we have to determine the 'loop-back', i.e. the response of small scales to large-scale structure changes.

\subsection{Influence of large-scale structures on small-scale turbulence}

We now concentrate on the small-scale wave turbulence described by $\mathbf{k}$ (where, as before, $|\mathbf{k}| \gg|\mathbf{q}|$ ), and consider the evolution of this microturbulence in a medium which is slowly modulated by large-scale structures. This can conveniently be described by the help of a wave kinetic equation for the wave action density $N_{k}(\mathbf{r}, t)$ in $\mathbf{r}-\mathbf{k}$ space [23]. As already stated, the standard expression for the wave action, $N_{k}=E / \omega_{k}$ (where $E$ is the wave energy), is not suitable for a system with mean flow $[24,25]$. Indeed, the latter expression can only be used when the total energy is contained in the small-scale waves. However, (3.1) shows clearly that the total energy is conserved and is composed of the part stored in small-scale and largescale structures. Thus we deal with a coupled system of two different parts of the same wave spectrum and, owing to the generation of large-scale fields, one cannot 
consider the energy being stored in the microturbulence only. Hence a new method has to be used.

The most consistent way of finding a generalized wave action $N_{k}$ is first to introduce a useful combination of field perturbations, a so-called 'normal variable' $[36]$

$$
\psi_{k} \equiv T_{k}+\delta B_{k}
$$

where $\delta$ is a coupling coefficient to be determined.

Starting again from the model equations (2.4) together with the Fourier expansion (3.2), but now looking for the set of equations for the small-scale quantities, we obtain

$$
\begin{array}{r}
\frac{\partial T_{k}}{\partial t}+i \alpha k_{y} B_{k}=-\frac{e \lambda^{2}}{2 m}(\mathbf{k} \times \mathbf{q}) \cdot \mathbf{z}\left(B_{q} T_{k-q}-B_{k-q} T_{q}\right), \\
\frac{\partial B_{k}}{\partial t}+\frac{i \beta k_{y}}{1+k^{2} \lambda^{2}} T_{k}=-\frac{e \lambda^{4}}{2 m}(\mathbf{k} \times \mathbf{q}) \cdot \mathbf{z} \frac{(k-q)^{2}-q^{2}}{1+k^{2} \lambda^{2}} B_{q} B_{k-q} .
\end{array}
$$

Multiplying (3.7b) by $\delta$, adding it to (3.7a) and assuming $q^{2} \lambda^{2} \ll 1$ yields

$$
\frac{\partial \psi_{k}}{\partial t}+i \omega_{k} \psi_{k}=-\frac{e \lambda^{2}}{4 m}(\mathbf{k} \times \mathbf{q}) \cdot \mathbf{z} \quad \psi_{k-q}\left(B_{q} \frac{1+2 k^{2} \lambda^{2}}{1+k^{2} \lambda^{2}}-\frac{1}{\delta} T_{q}\right) .
$$

If one recalls the linear results $(2.5)$ and (2.6), one can show that $\delta=\alpha k_{y} / \omega_{k}=$ $\sqrt{\alpha\left(1+k^{2} \lambda^{2}\right) / \beta}$.

The terms on the right-hand side of (3.8) can be transformed to obtain the equation for small-scale fluctuations,

$$
\frac{\partial \psi_{k}}{\partial t}+i \omega_{k} \psi_{k}+\psi_{k-q}\left[B_{q}\left(P_{q, k}^{(1)}+P_{q, k}^{(2)}\right)-T_{q} P_{q, k}^{(3)}\right]=0
$$

where the following variables have been introduced:

$$
\begin{gathered}
P_{q, k}^{(1)} \equiv \frac{e \lambda^{2}}{4 m}(\mathbf{k} \times \mathbf{q}) \cdot \hat{\mathbf{z}} \frac{1}{1+k^{2} \lambda^{2}}, \\
P_{q, k}^{(2)} \equiv \frac{e \lambda^{2}}{4 m}(\mathbf{k} \times \mathbf{q}) \cdot \hat{\mathbf{z}} \frac{2 k^{2} \lambda^{2}}{1+k^{2} \lambda^{2}}, \\
P_{q, k}^{(3)} \equiv \frac{e \lambda^{2}}{4 m}(\mathbf{k} \times \mathbf{q}) \cdot \hat{\mathbf{z}} \frac{1}{\delta} .
\end{gathered}
$$

The influence of the large-scale fields on the small-scale structures is contained in the third term of this equation. In order to investigate the evolution of the wave spectrum, we now have to find the wave kinetic equation.

\subsection{The wave kinetic equation in the presence of mean 'flow'}

Let us now derive the equation describing the evolution of the wave spectrum, the wave kinetic equation. To this end, we multiply (3.9) by $\psi_{k^{\prime}}$ and add the similar equation obtained by reversing $k$ and $k^{\prime}$. If we consider the amplitudes $\psi_{k}, \psi_{k^{\prime}}$ varying slowly with time and a small imaginary contribution to $\omega_{k}$, denoted $\gamma_{k}$, and average the whole equation over the fast-small-scale perturbations $\left(\int \ldots d \mathbf{k} \equiv\right.$ $\langle\ldots\rangle)$, we obtain

$$
\frac{\partial}{\partial t}\left\langle\psi_{k} \psi_{k^{\prime}}\right\rangle+\frac{\partial \omega_{k}}{\partial \mathbf{k}} \frac{\partial}{\partial \mathbf{r}}\left\langle\psi_{k} \psi_{k^{\prime}}\right\rangle-\frac{\partial \omega_{k}}{\partial \mathbf{r}} \frac{\partial}{\partial \mathbf{k}}\left\langle\psi_{k} \psi_{k^{\prime}}\right\rangle-2 \gamma_{k}\left\langle\psi_{k} \psi_{k^{\prime}}\right\rangle+S_{1}+S_{2}=0,
$$


where

$$
\begin{aligned}
& S_{1} \equiv\left\langle\psi_{k-q} \psi_{k^{\prime}}\right\rangle\left[B_{q}\left(P_{q, k}^{(1)}+P_{q, k}^{(2)}\right)-T_{q} P_{q, k}^{(3)}\right], \\
& S_{2} \equiv\left\langle\psi_{k} \psi_{k^{\prime}-q}\right\rangle\left[B_{q}\left(P_{q, k^{\prime}}^{(1)}+P_{q, k^{\prime}}^{(2)}\right)-T_{q} P_{q, k^{\prime}}^{(3)}\right] .
\end{aligned}
$$

Let us now introduce the spectral Wigner function defined by

$$
I_{k}(\mathbf{r}, t) \equiv \int d^{2} \mathbf{p}\left\langle\psi_{k} \psi_{-k+p}\right\rangle e^{i \mathbf{p} \cdot \mathbf{r}} .
$$

We set $\mathbf{k}^{\prime}=-\mathbf{k}+\mathbf{p}$ in (3.11), multiply it by $e^{i \mathbf{p} \cdot \mathbf{r}}$ and integrate over $d^{2} \mathbf{p}$. Thus, we obtain the evolution equation for $I_{k}$

$$
\frac{\partial}{\partial t} I_{k}+\frac{\partial \omega_{k}}{\partial \mathbf{k}} \frac{\partial}{\partial \mathbf{r}} I_{k}-\frac{\partial \omega_{k}}{\partial \mathbf{r}} \frac{\partial}{\partial \mathbf{k}} I_{k}-2 \gamma_{k} I_{k}+S_{1}+S_{2}=0
$$

with $S_{1}$ and $S_{2}$

$$
\begin{gathered}
S_{1}=\int d^{2} \mathbf{p} e^{i \mathbf{p} \cdot \mathbf{r}}\left\langle\psi_{-k+p} \psi_{k-q}\right\rangle\left[B_{q}\left(P_{q, k-q}^{(1)}+P_{q, k-q}^{(2)}\right)-T_{q} P_{q, k-q}^{(3)}\right], \\
S_{2}=\int d^{2} \mathbf{p} e^{i \mathbf{p} \cdot \mathbf{r}}\left\langle\psi_{-k+p-q} \psi_{k}\right\rangle\left[B_{q}\left(P_{q,-k+p}^{(1)}+P_{q,-k+p}^{(2)}\right)-T_{q} P_{q,-k+p}^{(3)}\right] .
\end{gathered}
$$

These quantities must now be calculated. Remembering that $|\mathbf{k}| \gg|\mathbf{q}|$, one can expand $I_{k-q}$ around $\mathbf{q}=0$, which leads to

$$
S_{1}=\left(I_{k}(\mathbf{r}, t)-\mathbf{q} \frac{\partial I_{k}(\mathbf{r}, t)}{\partial \mathbf{k}}\right)\left[B_{q}\left(P_{q, k-q}^{(1)}+P_{q, k-q}^{(2)}\right)-T_{q} P_{q, k-q}^{(3)}\right] e^{i \mathbf{q} \cdot \mathbf{r}} .
$$

Within the calculations of $S_{2}$, one finds that the only contribution is for the case $p=0$, i.e. $k^{\prime}=-k$, and one can therefore expand $P_{q,-k+p}$ around $p=0$ and thus obtain

$$
S_{2}=-\left(I_{k}(\mathbf{r}, t) \Lambda_{q, k}+i \frac{\partial \Lambda_{q, k}}{\partial \mathbf{k}} \frac{\partial I_{k}(\mathbf{r}, t)}{\partial \mathbf{r}}\right) e^{i \mathbf{q} \cdot \mathbf{r}}
$$

where

Furthermore, if we define

$$
\Lambda_{q, k} \equiv B_{q}\left(P_{q, k}^{(1)}+P_{q, k}^{(2)}\right)-T_{q} P_{q, k}^{(3)} .
$$

$$
\Delta \equiv-i \Lambda_{q, k} e^{i \mathbf{q} \cdot \mathbf{r}}
$$

(3.14) becomes the wave kinetic equation

$$
\frac{\partial I_{k}}{\partial t}+\frac{\partial}{\partial \mathbf{k}}\left(\omega_{k}+\Delta\right) \frac{\partial I_{k}}{\partial \mathbf{r}}-\frac{\partial}{\partial \mathbf{r}}\left(\omega_{k}+\Delta\right) \frac{\partial I_{k}}{\partial \mathbf{k}}=2 \gamma_{k} I_{k} .
$$

We still need to find the exact expression for $\Delta$. Explicit calculation directly yields

where

$$
\Delta=\frac{1+2 k^{2} \lambda^{2}}{1+k^{2} \lambda^{2}} \mathbf{k} \cdot \mathbf{v}_{B}^{(q)}-\frac{1}{\sqrt{1+k^{2} \lambda^{2}}} \mathbf{k} \cdot \mathbf{v}_{T}^{(q)}
$$

and

$$
\mathbf{v}_{B} \equiv-\frac{e \lambda^{2}}{4 m}(\nabla B \times \hat{\mathbf{z}})
$$

$$
\mathbf{v}_{T} \equiv-\frac{e \lambda^{2}}{4 m} \sqrt{\frac{\beta}{\alpha}}(\nabla T \times \hat{\mathbf{z}})
$$


Note that the only contributions to all the above calculations are due to $p \approx 0$ and thus from the definition of the Wigner function (3.13), $I_{k} \sim\left|\psi_{k}\right|^{2}$. Let us then introduce the action-like invariant $N_{k} \equiv I_{k}$, i.e.

$$
N_{k}=\left|\psi_{k}\right|^{2}=4 \frac{\alpha}{\beta}\left(1+k^{2} \lambda^{2}\right)\left|B_{k}\right|^{2} .
$$

We finally obtain the wave kinetic equation for magnetic electron drift mode turbulence with slowly varying parameters due to large-scale fields

$$
\frac{\partial N_{k}}{\partial t}+\frac{\partial \omega_{k}^{\mathrm{NL}}}{\partial \mathbf{k}} \frac{\partial N_{k}}{\partial \mathbf{r}}-\frac{\partial \omega_{k}^{\mathrm{NL}}}{\partial \mathbf{r}} \frac{\partial N_{k}}{\partial \mathbf{k}}=2 \gamma_{k} N_{k}-S t\left(N_{k}\right) .
$$

The linear frequency entering this equation is modified in the presence of largescale fields. The cause of this is the Doppler shift induced by the (large-scale) 'flow' velocity. Therefore the nonlinear frequency

$$
\omega_{k}^{\mathrm{NL}} \equiv \omega_{k}^{\mathrm{Re}}+\Delta
$$

has been introduced.

The collisional term $S t\left(N_{k}\right)$ has been added and it symbolically accounts for the wave damping by linear and nonlinear mechanisms, as well as local wave interactions, and guarantees saturation in the absence of large-scale fields. It balances the linear growth rate on the right-hand side of (3.21) and its form is not important here.

\subsection{Shearing of small-scale turbulence by large-scale fields}

We now want to determine the dynamics of magnetic electron drift mode turbulence under the influence of shearing fields. In the course of this analysis we will use quasi-linear theory and show that the broad spectrum of large-scale structures regulates turbulence by the process of random shearing, which will be represented by a diffusion in $\mathbf{k}$-space.

The linear part of the frequency $\omega_{k}$ is given by

$$
\left(\omega_{k}^{\mathrm{Re}}\right)^{2}=k_{y}^{2} \frac{\alpha \beta}{1+k^{2} \lambda^{2}} \sim \frac{k_{y}^{2} \lambda^{2}}{1+\kappa^{2} \lambda^{2}} \kappa_{n}^{2} v_{t h}^{2}
$$

and it determines the group velocity

$$
v_{\mathrm{g}}=\frac{\lambda}{\sqrt{1+\lambda^{2} k^{2}}} \kappa_{n} v_{t h} .
$$

In the limit $k \lambda \ll 1$, the group velocity takes the form $v_{\mathrm{g}} \simeq \lambda \kappa_{n} v_{t h}$ and the nonlinear part becomes

$$
\Delta \simeq \mathbf{k} \cdot\left(\mathbf{v}_{B}-\mathbf{v}_{T}\right) \equiv \mathbf{k} \cdot \mathbf{v}_{\mathrm{f}},
$$

so that

$$
\omega_{k}^{\mathrm{NL}}=\mathbf{k} \cdot\left(\mathbf{v}_{\mathrm{g}}+\mathbf{v}_{\mathrm{f}}\right),
$$

where $\mathbf{v}_{\mathrm{f}}$ denotes the 'flow' velocity, which is assumed to be much smaller than the group velocity. Furthermore, we decompose the wave spectrum $N_{k}$ into an average and a perturbed value $N_{k}=N_{0}+\tilde{N}$, where these quantities take the form $N_{0}=N_{0}(t)$ and $\tilde{N} \sim \exp \left(-i \Omega^{N} t+i \mathbf{p} \cdot \mathbf{r}\right)$. The average $N_{0}$ evolves in time only and with a much larger time scale than the perturbed part, which additionally evolves in space as well. The evolution of the perturbed wave spectrum $\tilde{N}_{k}$ can be seen 
as a modulation of the amplitude by large-scale fields. Thus $\mathbf{p}$ is of the order of q used previously to describe the large-scale fields. Averaging (3.21) over the fast small spatial scales and assuming that the collision term $\operatorname{St}\left(N_{k}\right)$ balances the linear instability $2 \gamma_{k} N_{k}$ yields

$$
\frac{\partial N_{0}}{\partial t}-\overline{\frac{\partial}{\partial \mathbf{r}}\left(\mathbf{k} \cdot \mathbf{v}_{\mathrm{f}}\right) \frac{\partial \tilde{N}}{\partial \mathbf{k}}}=0 .
$$

The equation for the perturbed part can then be found by subtracting (3.25) from (3.21), and linearizing the dissipative terms,

$$
\frac{\partial \tilde{N}}{\partial t}+\mathbf{v}_{\mathrm{g}} \frac{\partial \tilde{N}}{\partial \mathbf{r}}-\frac{\partial}{\partial \mathbf{r}}\left(\mathbf{k} \cdot \mathbf{v}_{\mathrm{f}}\right) \frac{\partial N_{0}}{\partial \mathbf{k}}=-\gamma^{N} \tilde{N}
$$

Note that we assume damping of the wave spectrum owing to the generation of large-scale fields and the factor of two in (3.21) enters the linear damping rate. Introducing the form of $\tilde{N}$ mentioned above into the latter equation and solving the resulting algebraic equation yields

$$
\tilde{N}=\frac{\partial}{\partial \mathbf{r}}\left(\mathbf{k} \cdot \mathbf{v}_{\mathrm{f}}\right) \frac{\partial N_{0}}{\partial \mathbf{k}} \frac{i}{\Omega^{N}-\mathbf{p} \cdot \mathbf{v}_{\mathrm{g}}+i \gamma^{N}} .
$$

Defining the response function $R(\Omega, p) \equiv i /\left(\Omega^{N}-\mathbf{p} \cdot \mathbf{v}_{\mathrm{g}}+i \gamma^{N}\right)$, we find that (3.25) takes the form

$$
\frac{\partial N_{0}}{\partial t}-\overline{\frac{\partial}{\partial \mathbf{r}}\left(\mathbf{k} \cdot \mathbf{v}_{\mathrm{f}}\right) \frac{\partial}{\partial \mathbf{k}}\left[\frac{\partial}{\partial \mathbf{r}}\left(\mathbf{k} \cdot \mathbf{v}_{\mathrm{f}}\right) \frac{\partial N_{0}}{\partial \mathbf{k}} R(\Omega, p)\right]}=0 .
$$

The condition for zonal magnetic fields is $\mathbf{q}=(q, 0,0)$. To simplify calculation we introduce the 'flow vector'

$$
\bar{B} \equiv \frac{e \lambda^{2}}{4 m}\left(B_{q}-\sqrt{\frac{\beta}{\alpha}} T_{q}\right) e^{i \mathbf{q} \cdot \mathbf{r}} .
$$

Note the similarity to the normal variable (3.6) defined to describe the small scales. Together with (3.23) and (3.20), we obtain

$$
\frac{\partial}{\partial \mathbf{r}}\left(\mathbf{k} \cdot \mathbf{v}_{\mathrm{f}}\right)=-q^{2} k_{y} \bar{B} \hat{x}
$$

Finally, introducing this relation into (3.28) yields the diffusion equation in $\mathbf{k}$-space

$$
\frac{\partial N_{0}}{\partial t}-\frac{\partial}{\partial k_{x}}\left[D_{k_{x}} \frac{\partial N_{0}}{\partial k_{x}}\right]=0
$$

where we defined the diffusion coefficient

$$
D_{k_{x}} \equiv k_{y}^{2} q_{x}^{4} \frac{e^{2} \lambda^{4}}{16 m^{2}}\left(|B|^{2}+\frac{\beta}{\alpha}|T|^{2}\right) R(\Omega, p) .
$$

This means that there is a diffusion of small-scale turbulence in $\mathbf{k}$-space towards even smaller scales $\left(D_{k_{x}}>0\right)$, where it will finally disappear because of dissipation. All this is due to large-scale structures. However, we have to remember that it is the small-scale structures that generate the large-scale fields in the first place. So by destroying (shearing) the small-scale turbulence, the large-scale structures destroy their life support and will be diminished as well. A reduced large-scale field gives rise to the possibility of increased small-scale turbulence and the cycle starts again. 
We thus have a predator-prey-type behaviour exhibited by the system containing large-(predator) and small-(prey) scale structures.

Using the same approach as for zonal magnetic fields, in the limit for magnetic streamers, $\mathbf{q}=(0, q, 0)$, from (3.23) and (3.20) one obtains

$$
\frac{\partial}{\partial \mathbf{r}}\left(\mathbf{k} \cdot \mathbf{v}_{\mathrm{f}}\right)=-q^{2} k_{x} \bar{B} \hat{y}
$$

with the same definition of $\bar{B}$ as in (3.29). Finally, the diffusion equation for magnetic streamers is obtained from (3.28) as before,

$$
\frac{\partial N_{0}}{\partial t}-\frac{\partial}{\partial k_{y}}\left[D_{k_{y}} \frac{\partial N_{0}}{\partial k_{y}}\right]=0
$$

with the diffusion coefficient

$$
D_{k_{y}} \equiv k_{x}^{2} q_{y}^{4} \frac{e^{2} \lambda^{4}}{16 m^{2}}\left(\left|B_{q}\right|^{2}+\frac{\beta}{\alpha}\left|T_{q}\right|^{2}\right) R(\Omega, p) .
$$

This is exactly the same mechanism as used for zonal fields. It is interesting to note that the diffusion coefficient depends on the square of the large-scale field amplitude.

Now, let us investigate the other limit, $k \lambda \gg 1$. The nonlinear frequency takes another form in this limit and can be written as

$$
\omega_{k}^{\mathrm{NL}} \approx \omega_{k}^{\mathrm{Re}}+2 \mathbf{k} \cdot \mathbf{v}_{B} .
$$

This means that the temperature does not take part in the process of shearing. It is therefore not necessary to introduce the 'flow vector' $\bar{B}$. However, the rest of the calculations are very similar to the above case and one can easily show that we obtain the same diffusion equations (3.31) and (3.34) for zonal fields and streamers but now with slightly different diffusion coefficients.

For zonal magnetic fields, shearing is described by the diffusion coefficient

$$
D_{k_{x}} \equiv k_{y}^{2} q_{x}^{4} \frac{e^{2} \lambda^{4}}{4 m^{2}}\left|B_{q}\right|^{2} R(\Omega, p),
$$

and the result for magnetic streamers corresponds exactly to that obtained for the zonal fields (3.36), but with exchanged subscripts $y$ and $x$,

$$
D_{k_{y}} \equiv k_{x}^{2} q_{y}^{4} \frac{e^{2} \lambda^{4}}{4 m^{2}}\left|B_{q}\right|^{2} R(\Omega, p) .
$$

Note that, in contrast to the limit $k \lambda \ll 1$, the large-scale temperature amplitude does not appear in the diffusion coefficient, i.e. in the process of the shearing of small-scale turbulence by large-scale structures.

\section{Conclusions}

In this paper we have presented the self-consistent theory of interactions between large-scale fields and magnetic electron drift mode turbulence. The mechanism for the generation of mean 'flow' by turbulence is identified and elucidated.

It has been shown that small-scale turbulence drives large-scale fields via magnetic Reynolds stress. The generated large-scale structures in turn form an environment for the parent waves, providing the modulation and regulation of the turbulence dynamics. In order to investigate the evolution of the wave spectrum 
the wave kinetic equation was derived and an appropriate adiabatic invariant for small-scale turbulence in the presence of mean 'flow' was found.

It was finally shown that the presence of large-scale structures leads to the shearing of the turbulence. As a consequence, a predator-prey-like behaviour and regulation of large- and small-scale turbulence have been found.

\section{Acknowledgements}

The second author would like to acknowledge the hospitality of the Department of Astronomy and Space Physics during his stay at Uppsala University as well as the support and open-mindedness of Professor Laurent Villard and the Ecole Polytechnique Fédérale in Lausanne, Switzerland, in the frame of bilateral scientific exchange.

\section{References}

[1] Shukla, P. K., Yu, M. Y., Rahman, H. U. and Spatscheck, K. H. 1981 Phys. Rev. A 23 , 321.

[2] Diamond, P. H., Itoh, S.-I., Itoh, K. and Hahm, T. S. 2005 Plasma Phys. Control. Fusion 47, 35 .

[3] Hasegawa, A., Maclennan, C. and Kodama, Y. 1979 Phys. Fluids 22, 2122.

[4] Busse, F. 1994 Chaos 4, 123.

[5] Sagdeev, R., Shapiro, V. and Shevchenko, V. 1979 Sov. J. Plasma Phys. 4, 306.

[6] Beyer, P., Benkadda, S., Garbet, X. and Diamond, P. H. 2000 Phys. Rev. Lett. 85, 4892.

[7] Onishchenko, O., Pokhotelov, O., Sagdeev, R., Shukla, P. and Stenflo, L. 2004 Nonlinear Proc. Geophys. 11, 241.

[8] National Oceanography Centre, University of Southampton, England. http://www.soc.soton.ac.uk/JRD/SAT/Rossby/Rossbyintro.html.

[9] Shukla, P. K. and Stenflo, L. 2002 Eur. Phys. J. D 20, 103.

[10] Smolyakov, A., Diamond, P. and Malkov, M. 2000 Phys. Rev. Lett. 84, 491.

[11] Itoh, K., Hallatschek, K., Itoh, S.-I., Diamond, P. and Toda, S. 2005 Phys. Plasmas 12, 062303 .

[12] Reames, D. V., Meyer, J. P. and Rosenvinge, T. T. 1994 Astrophys. J. 90, 649.

[13] Biskamp, D. 1994 Phys. Rep. 237, 179, and references therein.

[14] Vainshtein, S. I. and Cattaneo, F. 1992 Astrophys. J. 393, 165.

[15] Longcope, D. W. and Sudan, R. N. 1994 Astrophys. J. 437, 491.

[16] Aly, J. J. 1992 Plasma Phys. Control. Fusion 34, 1785.

[17] Holland, C. and Diamond, P. 2002 Phys. Plasmas 9, 3857.

[18] Smolyakov, A., Diamond, P. and Kishimoto, Y. 2002 Phys. Plasmas 9, 3826.

[19] Tatarakis, M. et al. 2002 Phys. Plasmas 9, 2244.

[20] Liu, S. Q. and Li, X. Q. 2000 Phys. Plasmas 7, 3405.

[21] Yu, M. Y., Shukla, P. K. and Stenflo, L. 1987 Phys. Fluids 30, 3299.

[22] Stenflo, L., Yu, M. Y. and Shukla, P. K. 1990 Phys. Fluids B 2, 1083.

[23] Vedenov, A., Gordeev, A. and Rudakov, L. 1967 Plasma Phys. 9, 719.

[24] Lebedev, V., Diamond, P., Shapiro, V. and Soloviev, G. 1995 Phys. Plasmas 2, 4420.

[25] Smolyakov, A. and Diamond, P. 1999 Phys. Plasmas 6, 4410.

[26] Tidman, A. and Shanny, R. 1974 Phys. Fluids 17, 1207.

[27] Jones, R. 1983 Phys. Rev. Lett. 51, 1269.

[28] Yu, M. and Stenflo, L. 1985 Phys. Fluids 28, 3447.

[29] Stenflo, L., Shukla, P. K. and Yu, M. Y. 1987 Phys. Rev. A 36, 955. 
[30] Nycander, J., Pavlenko, V. and Stenflo, L. 1987 Phys. Fluids 30, 1367.

[31] Pavlenko, V. and Uby, L. 1993 Phys. Fluids B 5, 1980.

[32] Nycander, J. and Pavlenko, V. 1991 Phys. Fluids B 3, 1386.

[33] Pavlenko, V. and Uby, L. 1994 Phys. Plasmas 1, 2140.

[34] Andruschenko, Z. and Pavlenko, V. 2004 Phys. Plasmas 11, 1402.

[35] Diamond, P. and Kim, Y.-B. 1991 Phys. Fluids B 3, 1626.

[36] Smolyakov, A. I., Diamond, P. H. and Medvedev, M. V. 2000 Phys. Plasmas 7, 3987. 